\title{
Questionnaire in French
}

\section{Risque Cardio Métabolique}

Date : I__ _ I _ _ I

$\begin{array}{ll}\text { Diabète } \square \text { oui } \square \text { non } & \text { Si oui DT2 }: \square \text { oui } \square \text { non } \\ & \text { Ancienneté du diabète }: / \_/ \text {ans }\end{array}$

\section{Paramètres démographiques}

Num : /

Groupe $: / \_$_ $\quad($ Cas $=1$ Témoin $=0) \quad($ cas $=$ ATCD de coronaropathie ou AVC $)$

Sexe : $\quad \square$ Masculin $\square$ Féminin (1=M, 2=F)

Date de naissance : /__ I_______ I

Lieu de naissance :

Code postal de résidence :

\section{Antécédents relatifs aux pathologies cardiovasculaires et artérielles.}

\section{Familiaux}

Familiaux d'HTA : $\square$ oui $\square$ non

Familiaux de diabète : $\square$ oui $\square$ non

\section{Personnels}

Hypertension : $\square$ oui $\square$ non

Personnel AVC : $\square$ oui $\square$ non

Coronaropathie : $\square$ oui $\square$ non

Infarctus : $\square$ oui $\square$ non

Angor : $\square$ oui $\square$ non

Artériopathie : $\square$ oui $\square$ non $\quad$ si oui, Amputation : $\square$ oui $\square$ non

Mode de vie

Tabac : $\square$ oui $\square$ non (oui=fumeur actuel ou arrêt depuis moins de 10 ans, non =les autres)

Alcool : $\square$ oui $\square$ non $\quad$ Type vin $\square$ oui $\square$ non rhum $\square$ oui $\square$ non si oui nbr verres/jour /__

Pratique sportive : $\square$ oui $\square$ non si oui nbr heures hebdomadaires /__

\section{$\underline{\text { Traitement en cours }}$}

Hypotenseur : $\square$ oui $\square$ non 
IEC : $\square$ oui $\square$ non $\quad$ Inhibiteurs calciques : $\square$ oui $\square$ non

Bétabloquants : $\square$ oui $\square$ non $\quad$ Autres Tt : $\square$ oui $\square$ non si oui type Tt :

Antidiabétiques :

ADO $\square$ insuline $\square$ les deux

Hypolipémiant: $\square$ oui $\square$ non

Antiagrégants plaquettaires : $\square$ oui $\square$ non

Données cliniques

Poids : $\quad / \quad / \mathrm{kg} \quad$ Taille $: \quad / \quad / \mathrm{m} \quad$ Tour de taille $/ \_/ \mathrm{cm}$

\section{Electrocardiogramme}

ECG : $\quad \square$ normal $\square$ anomalies

Si anomalie : ischémiques $\square$ oui $\square$ non

\section{Données biologiques}

CRP : /_ / $\mathrm{mg} / \mathrm{L}$

Hémoglobine : /__ / g/d

Urée : $\quad / \quad / \quad \mathrm{mmol} / \mathrm{L}$

Hématocrite : /__ / \%

Cholestérol total : / / $\mathrm{mmol} / \mathrm{L}$

Créatinine : $\quad / \_\_\mu \mathrm{mol} / \mathrm{L}$

HDL cholestérol : / / $\mathrm{mmol} / \mathrm{L}$

LDL cholesterol : / _ / $\mathrm{mmol} / \mathrm{L}$

Triglycérides : / _ / $\mathrm{mmol} / \mathrm{L}$

Glycémie : /__ / $\mathrm{mmol} / \mathrm{L}$ 